\title{
Scheduling jobs strategies for grid computing: A review
}

\author{
Salim Amdani" and Satish Jadhao \\ Babasaheb Naik College of Engineering \& Pusad, Maharashtra, India
}

\section{(C2016 ACCENTS}

\begin{abstract}
Distributed computing environments have been very much in spectacle for the last one and a half decade. But while widely used high performance computing platforms for scheduling of jobs becomes crucial due to the job starvation and resource fragmentations problems which exist in existing scheduling strategies. In the Meanwhile, these existing strategies are rarely balance fairness and performance aiming at these issues; we reviewed lot of literature and closely observed and tried to analyze the best and bad in of various methods available. A lot work has been done towards to promote job response time and throughput but they are not able to handle the "starvation" problem and lead to unfairness. However, fairness and performance in the cluster depiction is rarely taken into consideration simultaneously.
\end{abstract}

\section{Keywords}

Grid computing, Scheduling, Backfilling, Parallel computing, Distributed computing.

\section{Introduction}

The Grid concept was firstly introduced by Foster and Kesselman [1] [2] who defined it as a distributed and decentralized computer environment composed of large number of heterogeneous resources which are managed by different owners such as companies, universities or another business or scientific organisations [1].The difference between the conventional high performance computing such as cluster computing and grid is that grid tends to be heterogeneous and geographically distributed, loosely coupled. Job scheduling and resource scheduling are the two main necessities in grid computing. In job scheduling, the job scheduler has to find the appropriate resource for the job that the user submits [2]. Scheduling in Grid environments is significantly complicated due to the heterogeneous and dynamics nature of Grids. To overcome the heterogeneous and dynamic nature of Grids, the information service plays a highly important role. Since Grid computing is still emerging, the concept of Grid computing itself is evolving [3].Different scheduling policies can result in different user experiences and resource utilization. To promote job response time and throughput of the system, but they are not able to handle starvation problem and lead to unfairness. The fairness means that no job is delayed by any jobs of lower priority.

*Author for correspondence

82
Backfilling [4] [5] was proposed to help improve system utilization [1] and had been implemented in most production schedulers [6]. Nowadays, a research direction focuses on providing fair scheduling [4] between users in clusters. Backfilling [7] algorithm leverages fairness and performance in a simple and efficient manner [8]. Our paper makes two main contributions: 1) We illustrate the problem of job starvation and 2) resource fragmentations in traditional scheduling methods [6]. The rest of the paper is organized as follows. Section 2 reviews the related works. Then we analyze the problems of job starvation and resource fragmentations in section 3 .

\section{Related work}

Yi et.al. [9] proposed Combinational Backfilling Algorithm for parallel job scheduling in clusters. Krzysztof Kurowski et al. [10] proposed a new hierarchical scheduling strategy combining features of LB and FCFS with backfilling which dynamically groups ARs and does not require a static partitioning of computing resources. Suresh et.al.[11] Parallel job scheduling strategies like EASY, conservative backfill algorithms are failed to fill the resource gap fully Hafshejani et al. [12] developed a new method for efficient allocation of submitted jobs to resources. Jobs consist of threads that are arranged in a twodimensional matrix.

Simulation results of new scheduling method show quicker cluster system response time than FCFS and Backfilling scheduling methods. 
1) Advantage: Its advantage is that wasting resources is minimal and the use of resources is most.

2) Disadvantage: but there is some disadvantage such as it is possible that response time of a job is more than before specially for jobs at the first of queue or it is possible that primary-submitted jobs are not respond immediately. But still has the wastage and have chance for improvement in resource usage and response time. Reshmi et al. [13] discussed about various approaches of scheduling such as first systematic approach to scheduling problem was undertaken in the mid of 1950s, second half of the seventies, Scheduling the execution of parallel algorithms on parallel computer is an important and challenging area in current research. In parallel computation, the scheduling and the mapping tasks are considered to be the most critical problem. Shahid et al. [14] True benefits of the system are attained only when there is a proper mapping between hardware and software parallelism by preserving precedence and Communication requirements. The proposed strategy presented a scheduling model for a computational grid with the objective of minimizing the job TAT. The model searches for the best cluster and the best nodes for the job modules with respect to the execution time and specialization of the job. Yuan et al. [15] was proposed a new pre-emptive venture EASY backfilling (PVEASY) strategy, which integrates novel shadow load pre-emption (SLP) and venture backfilling (VB) approaches. To enhance the performance of EASY, many variants have been proposed, and most of them can be classified into two categories according to their changes:

1) Variants of Reservation Calculation;

2) Variants of Backfill Selection. Fairness and performance are two most important metrics of parallel job scheduling. Unfortunately, EASY and its variants still suffer from some serious problems that will be analysed in this section.

Qian Li et.al. [17] Proposed a reservation based FirstFit priority (R-FirstFit) job scheduling strategy for cluster rendering system. In this strategy, the reservation based FirstFit method is used to maximize. Moin Hasan et.al [16] an idea is presented to extend the CCS framework for the execution of the tasks based on their priority in the distributed environment. The concept of task reconsideration proposed for the CCS can be justified because already the techniques like EASY [8] and PVEASY [9] have been applied to backfill the low priority tasks. Qian $\mathrm{Li}$ et.al. [17] a novel hierarchal scheduling strategy for cluster interpretation system is proposed. The reservation based FirstFit (RF) job scheduling strategy and feedback based distribution for tasks (FD) strategy is together integrated to fulfil the maximization of system performance and load balancing. But Backfilling need the prediction by users or system, sometimes the prediction is not precisely, therefore backfilling would suffer from unfairness [15] and increase the blocked time of the system. Other job scheduling techniques have been designed to reduce the turnaround time for uses [18] and increase utilization in a fair environment. These analyses motivate us to propose a more powerful parallel job scheduler to guarantee strict fairness and employ prediction. What is the guaranteed fairness in parallel job scheduling? FCFS is an absolutely fair scheduling strategy, which holds the view that users are sensitive to service sequences, and jumping in a queue is unacceptable. Around 20 percent of the blocked jobs in each workload suffered from unfairness (delayed by later jobs, denoted as delay jobs).

\section{Research issues}

1) Job Starvation Problem: In recent paper [6] in First Fit job scheduling strategy, if there are many jobs which need fewer resources in the jobs queue and when the rendering system has idle CPUs, these idle CPUs can be used for the above jobs. But other jobs with high resource demand will not be executed as time goes by. In other words, the execution of jobs which need more resources will be delayed by the jobs which need less resources and may be never be executed as long as there still exist the jobs with low resource demand in the queue. That is the starvation phenomenon demonstrated in Figure 1. The horizontal axis represents the execution time of job and the vertical axis means the number of CPUs required. Figure $l(a)$ is the initialization state of scheduling. Job1 and Job2 is the running Job, Job3, Job4, Job5 and Job6 is the job of waiting for scheduling. Job3 is located on the head position of the job queue waiting for scheduling and Job3 has the higher resources demand than other jobs. According to the First Fit job scheduling strategy as shown in Figure 1(b), Job4, Job5 and Job6 will be executed on time T1, T2 and T3, respectively. And Job3 would not be executed for the high resources demand if the jobs queue exists the jobs with low resources demand such as Job4, Job5 and Job6, i.e., Job3 becomes starvation job. Actually, the strategy of resource reservation can be avoided the starvation of jobs and its basic idea is as follows. When the waiting time of the job in the awaiting for scheduling queue exceeds the waiting time threshold setting in advance, the 
system will obligate the resources and these resources cannot be allocated to other jobs. Until the idle reservation resources meet the resources requirement of reservation job, the resources will be allocated. And the problem of job starvation can be resolved.

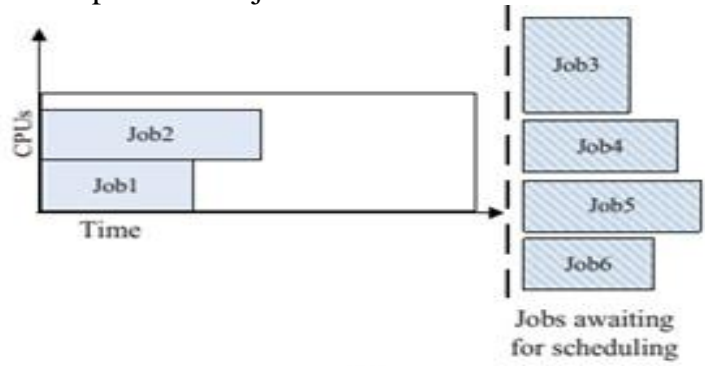

(a)

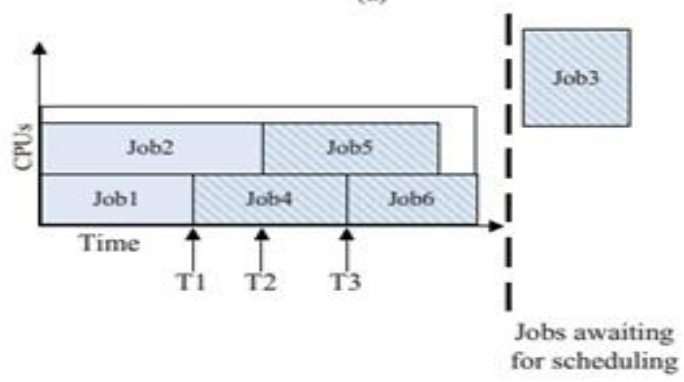

(b)

Figure 1 Procedure of job starvation phenomenon

2) Problem of resource fragmentations: The procedure of resource reservation strategy can resolve the job starvation, but it brings the problem of resource fragmentation demonstrated in Figure 2. The initialization state of scheduling is shown in Figure 2(a). Job1, Job3 and Job5 are the running job, Job2, Job4 and Job6 is the job of awaiting for scheduling, respectively. Job2 is located on the head position of the job queue awaiting for scheduling. According to the resource reservation strategy, the scheduler will reserve the resources for Job2. As the reserved CPUs cannot be used by other jobs, Job4 and Job6 will not be scheduled on time T1. On time T2, the idle CPUs can meet the resources demand of Job2 and then Job2 will be executed. From the Figure 2(b), there are three resource fragmentations, i.e., frag 1 , frag 2 and frag 3 , and frag2 is produced by the resource reservation.

These resource fragmentations lead to the idle of resources and the decline of resources utilization rate. In order to deal with masses of rendering jobs and reduce the waiting time of users jobs submitted, an efficient scheduling method should be able to avoid job starvation and resource fragmentations and maximize system throughput.

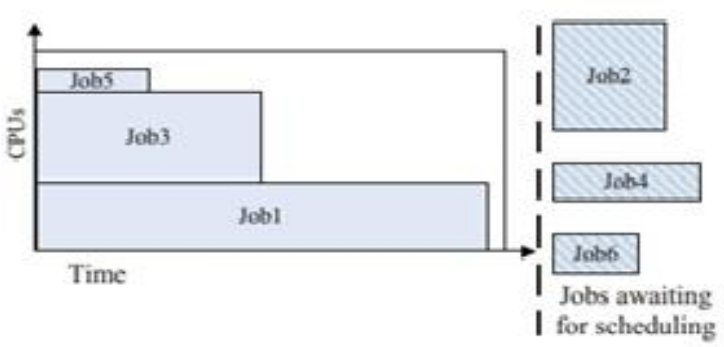

(a)

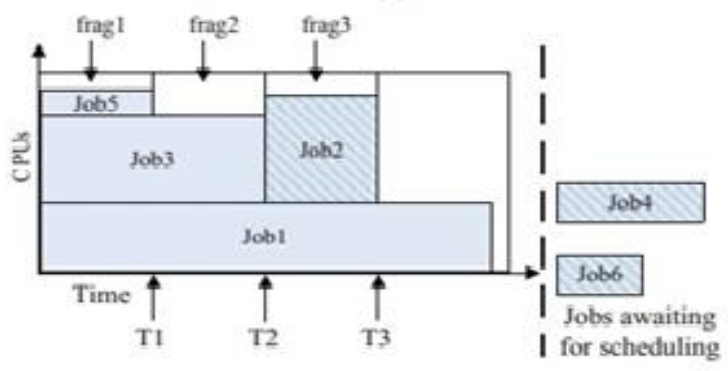

(b)

Figure 2 Procedure of resource fragmentations

\section{Conclusion}

We characterize the job starvation and resource fragmentations problem in the process of scheduling jobs strategies. Hence it is required a efficient method to address these issues more closely and fairly so that we can utilize Grid Computing resource in an efficient and fair manner. It has a scope of multifaceted options to improve the performance of the system.

\section{Acknowledgment}

None.

\section{Conflicts of interest}

The authors have no conflicts of interest to declare.

\section{References}

[1] Baraglia R, Dazzi P, Capannini G, Pagano G. A multicriteria job scheduling framework for large computing farms. In IEEE $10^{\text {th }}$ international conference on computer and information technology (CIT) 2010 (pp. 187-94). IEEE.

[2] Dongarra J, Lastovetsky A. An overview of heterogeneous high performance and grid computing. Engineering the Grid: Status and Perspective. 2006:125.

[3] Foster I, Zhao Y, Raicu I, Lu S. Cloud computing and grid computing 360-degree compared. In grid computing environments workshop 2008 (pp. 1-10). IEEE.

[4] Doulamis ND, Doulamis AD, Varvarigos EA, Varvarigou TA. Fair scheduling algorithms in grids. IEEE Transactions on Parallel and distributed systems. 2007; 18(11):1630-48. 
[5] Lokhande SF, Chavhan SD, Jadhao SR. Grid computing scheduling jobs based on priority using backfilling. IJEECSE. 2015; 2(2):68-72.

[6] Li Q, Wu W, Zhou X, Sun Z, Huang J. R-FirstFit: a reservation based first fit priority job scheduling strategy and its application for rendering. In IEEE $17^{\text {th }}$ international conference on computational science and engineering (CSE) 2014 (pp. 1078-85). IEEE.

[7] Jadhao SR, Amdani SY. Job scheduling using backfilling techniques in grid computing: a survey. In international conference on advances in computing and information technology (ICACIT) 2014(pp. 110 4). Elsevier.

[8] Yuan Y, Yang G, Wu Y, Zheng W. PV-EASY: a strict fairness guaranteed and prediction enabled scheduler in parallel job scheduling. In proceedings of the $19^{\text {th }}$ ACM International symposium on high performance distributed computing 2010 (pp. 240-51). ACM.

[9] Yi S, Wang Z, Ma S, Che Z, Liang F, Huang Y. Combinational backfilling for parallel job scheduling. In $2^{\text {nd }}$ International conference on education technology and computer (ICETC) 2010 (pp.112-6). IEEE.

[10] Kurowski K, Oleksiak A, Piątek W, Węglarz J. Hierarchical scheduling strategies for parallel tasks and advance reservations in grids. Journal of scheduling. 2013; 16(4):349-68.

[11] Suresh A, Vijayakarthick P. Improving scheduling of backfill algorithms using balanced spiral method for cloud metascheduler. In international conference on recent trends in information technology (ICRTIT) 2011 (pp. 624-7). IEEE.

[12] Hafshejani ZM, Mirtaheri SL, Khaneghah EM, Sharifi M. An efficient method for improving backfill job scheduling algorithm in cluster computing systems. The International Journal of Soft Computing and Software Engineering. 2013; 3(3): 422-9.

[13] Reshmi R, Shanthi D. Batch scheduling based on QOS in HPC-A survey. International Journal of Networking \& Parallel Computing. 2013; 2(2): 1-8.

[14] Shahid M, Raza Z. A precedence based distributed job scheduling for computational grid. In IEEE international advance computing conference (IACC) 2014 (pp. 702-7). IEEE.
[15] Yuan Y, Wu Y, Zheng W, Li K. Guarantee strict fairness and utilize prediction better in parallel job scheduling. IEEE Transactions on Parallel and Distributed Systems. 2014; 25(4):971-81.

[16] Hasan M, Goraya MS. A framework for priority based task execution in the distributed computing environment. In international conference on signal processing, computing and control (ISPCC) 2015 (pp. 155-8). IEEE.

[17] Li Q, Wu W, Sun Z, Wang L, Huang J, Zhou X. A novel hierarchal scheduling strategy for rendering system. In 2015 international conference on identification, information, and knowledge in the internet of things (IIKI) 2015 (pp. 206-9). IEEE.

[18] Thebe O, Bunde DP, Leung VJ. Scheduling restartable jobs with short test runs. In JSSPP 2009 (pp. 116-37).

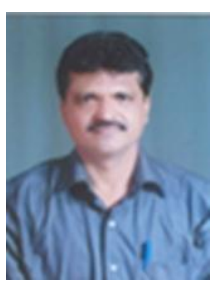

Dr. S.Y. Amdani received his Ph.D.(CSE) degree from SGB Amravati University ,Amravati and Working As Head and Professor in Deptt. Of CSE at B.N.C.E.Pusad(india), and Life member of Indian Society for Technical Education,New Delhi.He has published and presented may research papers in International conferences and journals.He is also Editorial Board member of many international journals.

Email: salimamdani@yahoo.com

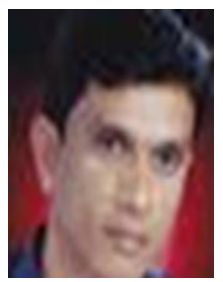

Prof. S.R. Jadhao is working as an Assistant Professor in Deptt. Of CSE at B.N.C.E.Pusad(india), and Life member of Indian Society for Technical Education,New Delhi.He has published and presented may research papers in International conferences and journals.He is specilized in Design and Analysis of Algorithm(DAA),JAVA,J2EE and Grid Computing. 